\title{
Development of a Realistic Set of Synthetic Earth Impactor Orbits
}

\section{Chesley, Steven R.}

IEEE

2019

Chesley, S R , Valsecchi , G B , Eggl , S , Granvik , M , Farnocchia , D \& Jedicke , R 2019 , Development of a Realistic Set of Synthetic Earth Impactor Orbits . in 2019 IEEE

AEROSPACE CONFERENCE . IEEE Aerospace Conference Proceedings, IEEE , IEEE Aerospace Conference, Big Sky, MT , United States , 02/03/2019 . https://doi.org/10.1109/AERO.2019.8742172

http://hdl.handle.net/10138/325473

https://doi.org/10.1109/AERO.2019.8742172

unspecified

acceptedVersion

Downloaded from Helda, University of Helsinki institutional repository.

This is an electronic reprint of the original article.

This reprint may differ from the original in pagination and typographic detail.

Please cite the original version. 


\section{Development of a Realistic Set of Synthetic Earth Impactor Orbits}

\author{
Steven R. Chesley \\ Solar System Dynamics Group \\ Jet Propulsion Laboratory \\ California Institute of Technology \\ Pasadena, CA 91109 \\ steve.chesley@jpl.nasa.gov \\ Mikael Granvik \\ Dept. of Computer Science, \\ Electrical \& Space Engr. \\ Luleå University of Technology \\ S-98128 Kiruna, Sweden \\ Dept. of Physics \\ University of Helsinki \\ FI-00014 Helsinki, Finland \\ mgranvik@iki.fi
}

\author{
Giovanni B. Valsecchi \\ IAPS-INAF \\ 00133 Rome, Italy \\ IFAC-CNR \\ 50019 Sesto Fiorentino, Italy \\ giovanni.valsecchi@inaf.it \\ Davide Farnocchia \\ Solar System Dynamics Group \\ Jet Propulsion Laboratory \\ California Institute of Technology \\ Pasadena, CA 91109 \\ davide.farnocchia@jpl.nasa.gov
}

\author{
Siegfried Eggl \\ LSST/DIRAC Institute \\ Astron. and Astroph. Dept. \\ University of Washington \\ Seattle, WA 98105 \\ eggl@uw.edu \\ Robert Jedicke \\ Institute for Astronomy \\ University of Hawaii \\ Honolulu, HI 96822 \\ jedicke@hawaii.edu
}

\begin{abstract}
We present a refined method for creating orbits of fictitious Earth impactors that are representative of the actual impactor population. Such orbits are crucial inputs to a variety of investigations, such as those that seek to discern how well and how early a particular asteroid survey can detect impactors, or to understand the progression of impact probability as an object is tracked after discovery. We will describe our method, which relies on Öpik's b-plane formalism, and place it in context with previous approaches. While the Öpik framework assumes the restricted three body problem with a circular Earth orbit, our final synthetic impactors are differentially corrected to ensure an impact in the N-body dynamics of the solar system. We also test the validity of the approach through brute force numerical tests, demonstrating that the properties of our synthetic impactor population are consistent with the underlying NearEarth Object (NEO) population from which it is derived. The impactor population is, however, distinct from the NEO population, not only by virtue of the proximity of the asteroid orbit to that of the Earth, but also because low encounter velocities are strongly favored. Thus the impacting population has an increased prominence of low inclination and low eccentricity orbits, and Earth-like orbits in particular, as compared to the NEO population as a whole.
\end{abstract}

\section{TABle of Contents}

1 InTRODUCTION $\ldots \ldots \ldots \ldots \ldots \ldots \ldots \ldots \ldots \ldots \ldots \ldots$

2 Computing neO Impact Probability ...... 2

3 DERIVING IMPACTORS FROM NEOS .......... 3

4 Properties of the Impacting Population 5

5 Conchusion .......................... 6

ACKNOWLEDGMENTS $\ldots \ldots \ldots \ldots \ldots \ldots \ldots \ldots \ldots .6$

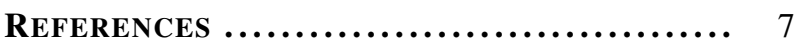

BIOGRAPHY $\ldots \ldots \ldots \ldots \ldots \ldots \ldots \ldots \ldots \ldots, 7$

\section{INTRODUCTION}

The Earth orbits amongst a cloud of small rocky asteroids that routinely approach the planet, often very closely, sometimes even colliding. This, of course, can be a serious problem to

978-1-5386-6854-2/19/\$31.00 (C)2019 IEEE those of us who happen to inhabit the Earth, but fortunately the most frequent impactors are the smallest asteroids, with the least ability to wreak damage on the surface.

Our questions are, what can be said about the orbital characteristics of Earth impactors? In what ways are their orbits substantially different from the NEO population as a whole? What do these differences imply for the Earth hazard problem? And can this information be exploited to develop superior asteroid search strategies?

With the near-Earth object (NEO) catalog now containing over 19,000 objects and the catalog increasing by about 2000 discoveries per year, we have a remarkably detailed understanding of the orbital distribution of the NEOs, including the characteristics of those not yet discovered. This knowledge allows us to generate a synthetic population of NEOs with the same properties as the real population. Our objective here is to start with such a synthetic NEO population and to derive from it a synthetic impactor population.

This objective has been tackled in the past, most notably by Chesley \& Spahr [1] and for the Synthetic Solar System Model published by Grav et al. [2], which utilized the Chesley $\&$ Spahr methodology. But we believe the time is right to revisit the problem. First, the Chesley \& Spahr work relied on the Bottke et al. NEO model [3], which was derived from only a few hundred discoveries obtained prior to 2000 . Now our knowledge of the NEO population is vastly superior to that available in 2000. Indeed, the recent NEO population model developed by Granvik et al. [4] utilizes thousands of asteroids from two different search instruments to obtain a far more refined and richer picture of the NEO population. Secondly, the impactor selection algorithm that we present here is substantially improved, both in speed and accuracy, over the one used by Chesley \& Spahr. As we shall see, the earlier work tended to overemphasize orbits with lower velocity encounters, and we believe that the present work provides a more accurate characterization of the impactor population.

In this paper we present an algorithm for generating an unbiased set of Earth-impacting orbits and we analyze some of the characteristics of this population. We start in Sec. 2 
with a brief introduction to some of the key elements of the Öpik theory of close approaches, including the calculation of the impact probability of an NEO. In Sec. 3 we describe in detail our algorithm for obtaining impactors. Sec. 4 presents an analysis of the orbital characteristics of the impacting population and makes a comparison with those of the broader NEO population.

\section{Computing NeO Impact Probability}

The impactor population is selected from among the NEO population according to each object's annualized impact probability. Objects that cannot impact because their orbits do not approach closely enough to the orbit have a zero impact probability, and so we are only concerned with those objects where the Minimum Orbital Intersection Distance (MOID) is near zero. In this scenario, Öpik's close approach theory [5] provides an excellent framework for estimating the annualized impact probability of a given asteroid. Here we provide a very concise discussion of the assumptions and tools of Öpik's theory. For a complete description and derivation see, e.g., [6].

\section{Öpik Theory - The Short Course}

We assume an asteroid encountering a fictitious Earth having zero inclination and eccentricity and semimajor axis at 1 au. Both objects follow two-body heliocentric orbits, save for an impulsive trajectory correction to the asteroid near the time of the encounter. The Öpik analysis uses a co-rotating reference frame having $X$ in the Sun-Earth direction, $Y$ in the direction of the Earth's heliocentric velocity vector and $Z$ in the direction of the Earth's orbital angular momentum. In this frame the unperturbed Earth-relative velocity of the asteroid at encounter is given by $\vec{U}=\left(U_{X}, U_{Y}, U_{Z}\right)$. These velocities can be expressed in terms of the asteroid elements by

$$
\begin{aligned}
& U_{X}= \pm \sqrt{2-\frac{1}{a}-a\left(1-e^{2}\right)} \\
& U_{Y}=\sqrt{a\left(1-e^{2}\right)} \cos i-1 \\
& U_{Z}= \pm \sqrt{a\left(1-e^{2}\right)} \sin i .
\end{aligned}
$$

Here, as is customary in Öpik formulae, the velocity is expressed in units of the Earth's heliocentric velocity, which in au/day is numerically equivalent to the Gauss gravitational constant $k$. The sign of the radial velocity $U_{X}$ depends on whether the close approach is taking place on the preperihelion $\left(U_{X}<0\right)$ or post-perihelion $\left(U_{X}>0\right)$ portion of the orbit. The sign of $U_{Z}$ is determined by the node at which the encounter takes place; $U_{Z}<0$ at the descending node and $U_{Z}>0$ at the ascending node.

In this representation we have

$$
\begin{aligned}
& U_{X}=U \sin \theta \sin \phi \\
& U_{Y}=U \cos \theta \\
& U_{Z}=U \sin \theta \cos \phi
\end{aligned}
$$

and, conversely,

$$
\begin{aligned}
\cos \theta & =\frac{U_{Y}}{U} \\
\tan \phi & =\frac{U_{X}}{U_{Z}} .
\end{aligned}
$$

The plane orthogonal to $\vec{U}$ with the geocenter at its origin is the key space for Öpik close encounter analyses. We use $\vec{b}$ to denote the vector in this plane from the origin to the intersection with the inbound asymptote of the asteroid. A convenient coordinate system for this so-called $b$-plane has the $\zeta$-axis oriented opposite the projection of the Earth's heliocentric velocity vector and the $\xi$-axis oriented to form a right-handed system $\xi-\eta$ - $\zeta$, where the $\eta$-axis is aligned with $\vec{U}$. That is, the unit vector in the direction of the $\xi$-axis is $\hat{\xi}=\hat{\eta} \times \hat{\zeta}$. The impact parameter is given by $b=\sqrt{\xi^{2}+\zeta^{2}}$.

With this formulation, $\xi$ is equivalent to the asteroid MOID, i.e., the closest that the asteroid can come to the geocenter, neglecting gravitational focusing, for any combination of Earth and asteroid anomaly. And $\zeta$ indicates how early or late the asteroid is for the minimum distance encounter, with a positive $\zeta$ indicating that the asteroid is late and negative when the asteroid is early. Gravitational focusing allows an impact when the asymptote is close enough to the figure of the Earth, even if does not intersect. The impact cross-section is defined by the circle $b<b_{\oplus}$ on the $b$-plane, where

$$
b_{\oplus}=r_{\oplus} \sqrt{1+\frac{V_{e}^{2}}{U^{2}}} .
$$

Here $V_{e}^{2}=2 G M_{\oplus} / r_{\oplus}$ refers to the Earth escape velocity, and $r_{\oplus}$ is the Earth radius, which should be enlarged by $40-$ $50 \mathrm{~km}$ to account for atmospheric capture. We assume $r_{\oplus}=$ $6420 \mathrm{~km}$ in this study.

\section{Impact Probability}

We intend to select our impactor population from among the NEA population according to a weighting that is proportional to the annualized impact probability. Within the Öpik setting we first compute the impact probability per asteroid revolution as the fraction of the Earth's orbital longitude $\lambda_{\oplus}$ for which an impact is possible:

$$
\mathrm{IP}_{\mathrm{rev}}=\frac{\delta \lambda_{\oplus}}{2 \pi}
$$

Now, from Öpik theory we have

$$
\delta \lambda_{\oplus}=\frac{\delta \zeta}{\sin \theta},
$$

where $\delta \zeta=2 \sqrt{b_{\oplus}^{2}-\xi^{2}}$ is the length of the chord within the impact cross-section at the associated value of $\xi$. To transform from the probability per asteroid revolution to the per annum probability we must divide by the asteroid period in years $P_{\text {ast }}=a^{\frac{3}{2}}$, where $a$ is the semimajor axis of the asteroid in au. Assembling the above we obtain the analytic formula for annualized impact probability

$$
\mathrm{IP}_{\mathrm{ann}}=\frac{\sqrt{b_{\oplus}^{2}-\xi^{2}}}{\pi a^{\frac{3}{2}} \sin \theta} .
$$

We note that this annualized impact probability for a fixed asteroid orbit is different from the impact probability usually attributed to Öpik. The classical Öpik impact probability is computed per asteroid revolution and is averaged over the precession period of the line of apsides, which is typically of order $10^{4}$ years. Of course, in the classical framework, 
there are four node crossings per precession period, and for most of the precession cycle impacts are not possible, thus the classical probabilities are far lower than the annualized probabilities for a non-precessing orbit with a MOID small enough to allow an impact.

\section{Monte Carlo validation}

As a validation of the computation of the impact probability given by Eq. 1, we implement a Monte Carlo test. Recalling the two-body nature of the problem to this point, suppose the asteroid is at the node where an impact can occur and the Earth is at an arbitrary position in its orbit. If we advance both the Earth and the asteroid by exactly one asteroid period then the asteroid will return to its previous position and the Earth may or may not be close to the asteroid. In the context of a mapping, the asteroid remains frozen at the node and the Earth advances in time by $P_{\text {ast }}$ and thus in longitude by $\Delta \lambda_{\oplus}=n_{\oplus} P_{\text {ast }}$, where $n_{\oplus}$ is the mean motion of the Earth. At each iteration where the Earth falls near the asteroid, we check if $b<b_{\oplus}$, in which case we count a Monte Carlo impact. After getting a sufficiently large number of impacts (100 in this study), the impact probability per asteroid revolution is the quotient of the number of impacts and the number of asteroid revolutions tested.

Figure 1 shows the results of this validation test for a sampling of NEAs for which $\xi<b_{\oplus}$, a prerequisite for an impact to be possible. The figure indicates a broad agreement, though there are a number of outliers that are traceable to near-resonant orbits. For objects in or near a mean motion resonance with Earth, the Monte Carlo method actually serves as a poor validation because the resonance prevents a uniform Monte Carlo sampling. Thus the outliers in Fig. 1 actually point to a deficiency in the Monte Carlo approach, and not the analytic Öpik approach. The conclusion from this short experiment is that the analytic approach is correct, and indeed more reliable than the Monte Carlo approach used as a validation.

\section{Relation to previous work}

We pause at this point to compare the impact probabilities from this work with those from Chesley \& Spahr [1]. Chesley $\&$ Spahr computed the impact probability as the fraction of a revolution that an NEO spent within the thin torus surrounding the Earth's orbit having radius $b_{\oplus}$. Put another way, the torus is given by the volume of space that is less than $b_{\oplus}$ from some point on the Earth's orbit. Here we use the term tubular impact probability to refer to this approach. We have here adopted an annualized impact probability, as is appropriate for our problem, and so our impact probabilities immediately differ from those of Chesley \& Spahr by a factor equal to the orbital period in years, i.e., by $a^{\frac{3}{2}}$ with $a$ in au. Additionally, even after this correction, the Chesley \& Spahr impact probabilities were significantly higher at low $V_{\infty}$ and lower at high $V_{\infty}$, which had the effect of increasing the presence of low $V_{\infty}$ in a subtle but significant way. This phenomenon can be clearly seen in Figure 2, which depicts the ratio between the annualized tubular impact probability and $\mathrm{IP}_{\text {ann }}$ as given in Eq. 1. The median $V_{\infty}$ reported by Chesley \& Spahr is about $10 \mathrm{~km} / \mathrm{s}$, whereas our new work suggests a value around $14 \mathrm{~km} / \mathrm{s}$, as we show below. Understanding and documenting the reason for these discrepancies is an area of continued investigation.
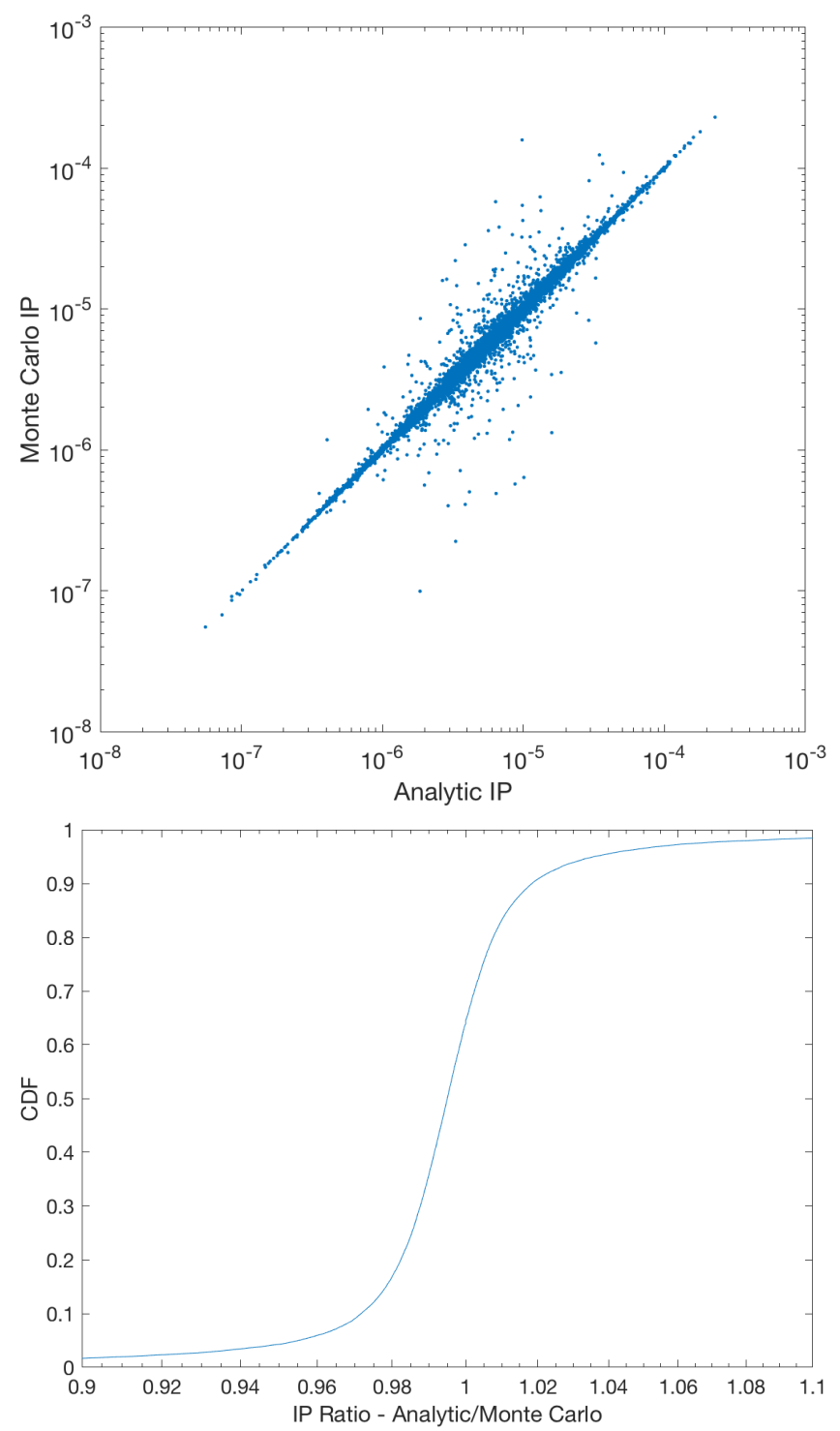

Figure 1. (top) Comparison between the Monte Carlo and analytic Öpik impact probabilities for a random sample of low-MOID NEAs. A small fraction of Monte Carlo values depart from the analytic due to orbital resonances, which corrupt the Monte Carlo result. (bottom) Cumulative distribution function of the ratio between the analytic and Monte Carlo impact probabilities. Approximately $5 \%$ of Monte Carlo cases differed from the analytic result by over $7 \%$.

\section{DERIVING IMPACTORS FROM NEOS}

Our approach to developing a synthetic Earth impactor population that matches the orbital properties of the true impactor population consists of four essential steps:

1. Sample a synthetic NEO population

2. Discard objects with orbits for which an impact is not possible

3. Subsample the remainder according to their impact probability

4. Adjust the orbits of the impactors to enforce an impact at a selected year

We note that steps 2 and 3 above are taken within the Öpik 


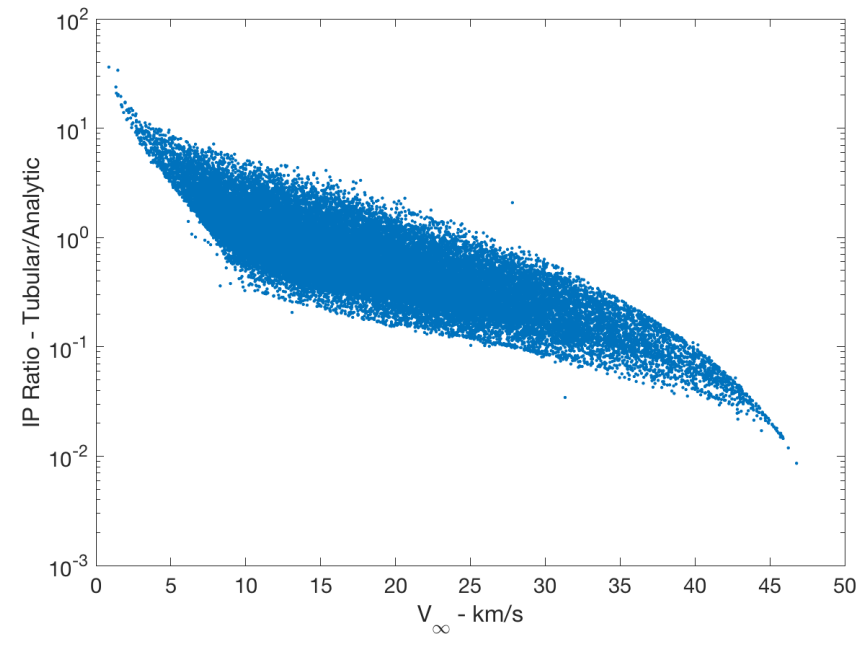

Figure 2. Ratio of tubular to analytic impact probabilities as a function of $V_{\infty}$.

framework, which assumes two-body dynamics and a circular Earth orbit. In the following we describe each of these procedures in more detail.

\section{Step 1. The NEO population}

A prerequisite for developing a true-to-life set of impactors is the availability of an accurate model of the NEO population. There is a long and rich history of efforts to debias the known NEO population to derive the size and orbital distribution of the actual NEO population. The current state of the art in this arena is captured by Granvik et al. [4], who have produced a detailed model for the orbital and absolute magnitude distribution of the NEO population. This model, which we refer to as the Granvik model, is the starting point for our impactor population.

In the Granvik model the NEO orbital distribution varies with the absolute magnitude $H$, which allows one to produce a debiased synthetic population of NEOs that correctly represents the number of objects in each $H$ bin. However, we want to capture the overall distribution of impacting orbits in each size bin, and so for our work we intend to produce thousands of synthetic impactors all at a given absolute magnitude. If we followed some realistic size distribution for the impactors then the bins at small $H$ (large size) would have only a few objects that might not be representative of the population as a whole. In this paper we consider only the smallest size bin in the Granvik model, $24.75<H<25$.

We use the traditional notation $(a, e, i, \Omega, \omega)$ to represent the orbital elements semimajor axis, eccentricity, inclination, longitude of ascending node and argument of perihelion, respectively. The Granvik model only specifies the correlated $(a, e, i)$ distribution for each $H$ bin in the model. We supplement that with $\Omega$ and $\omega$ drawn from a uniform distribution over their range $\left(0-360^{\circ}\right)$. Thus at this stage, until Step 4, each sampled NEO has five orbital elements specified.

\section{Step 2. Low-MOID Asteroids}

The procedure is to draw NEOs from the Granvik model and to test whether they have a MOID that is small enough to allow an Earth impact. If not then we discard the NEO and

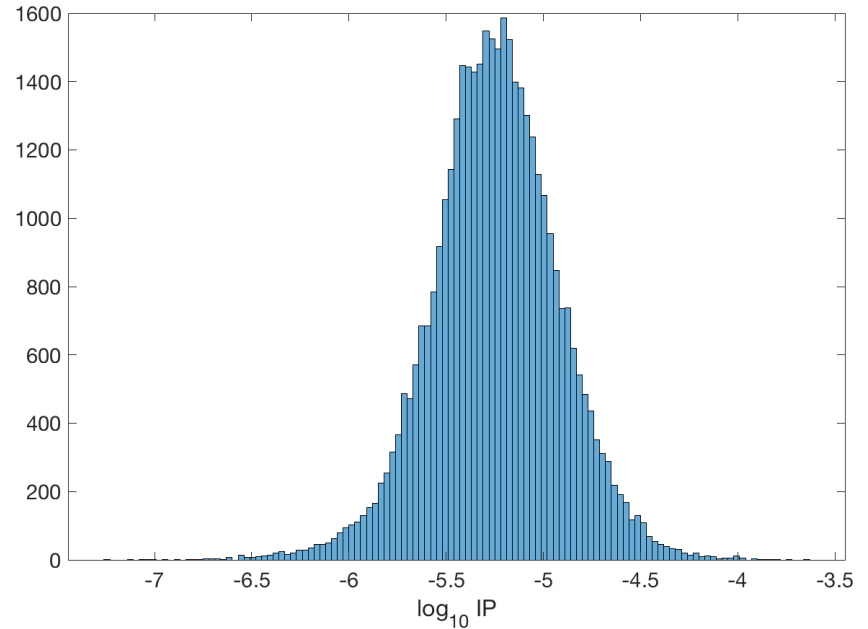

Figure 3. Distribution of impact probabilities $\mathrm{IP}_{\text {ann }}$ among 39,157 randomly selected LMAs. For every LMA in this plot there are approximately 4650 NEOs with zero impact probability that are not shown.

draw another. To this end we compute (see Ref. [6])

$$
\xi^{*}=\cos \phi\left[\frac{a\left(1-e^{2}\right)}{1+e \cos \omega}-1\right]
$$

Since $\xi^{*}$ is equal to the MOID, we can simply test $\xi^{*}<b_{\oplus}$ to verify that an impact is possible. Such objects, which we term Low-MOID Asteroids (LMAs), have at least the potential for impact. We note that the orbital properties of Potentially Hazardous Asteroids (PHAs), which have MOID $<0.05$ au, should be substantially the same as those of LMAs, and so we consider LMAs to be a suitable proxy for PHAs. For its smallest size bin, the Granvik model produces one LMA for every 4650 NEOs sampled.

\section{Step 3. Subsampling for Impactors}

If an object passes the screening in the previous step it is an LMA and so we calculate the impact probability $\mathrm{IP}_{\text {ann }}$ according to Eq. 1. The distribution of $\mathrm{IP}_{\text {ann }}$ is depicted in Fig. 3. We sample impactors from among the LMAs according to a scaled impact probability. Thus for each LMA, we select a random number $0<s \leq 1$ from a uniform distribution. If $s<\mathcal{S} \times \mathrm{IP}_{\text {ann }}$ then that LMA is retained as a member of our impacting population. Otherwise we record the LMA characteristics for later analysis and return to Step 1 to draw a new NEO. Here $\mathcal{S}$ is merely a scale factor that is used to increase the ratio of the number of impactors to LMAs without altering the final distribution.

From Fig. 3, it is clear that nearly all cases have $\mathrm{IP}_{\text {ann }}<$ $10^{-4}$. Indeed, only one in $\sim 2000$ LMAs have an impact probability that exceeds this value, and so we use $\mathcal{S}=10^{4}$. This value of $\mathcal{S}$ allows us to obtain one impactor for every 13 LMAs. When combined with the filtering in Step 2, we must sample $\sim 60,000$ NEOs to obtain one impactor.

\section{Step 4. Correcting for N-body effects}

Recalling that our synthetic impactors are derived from twobody dynamics with a circular Earth orbit, they are in fact only preliminary impact orbits. We must now ensure that the selected impactors are on an impact trajectory with an accurate dynamical model of the solar system. To this end 

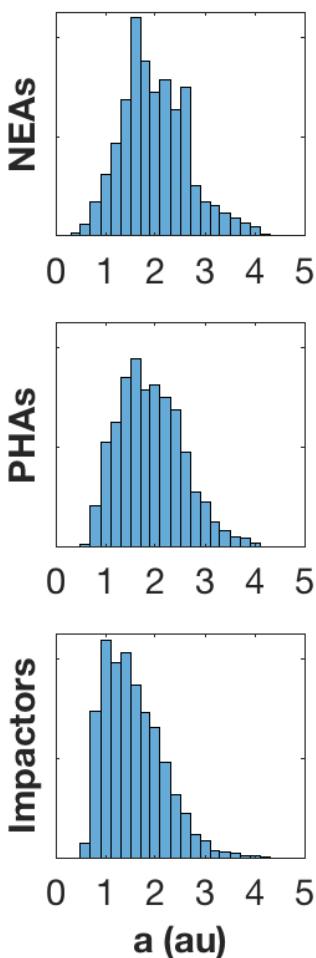
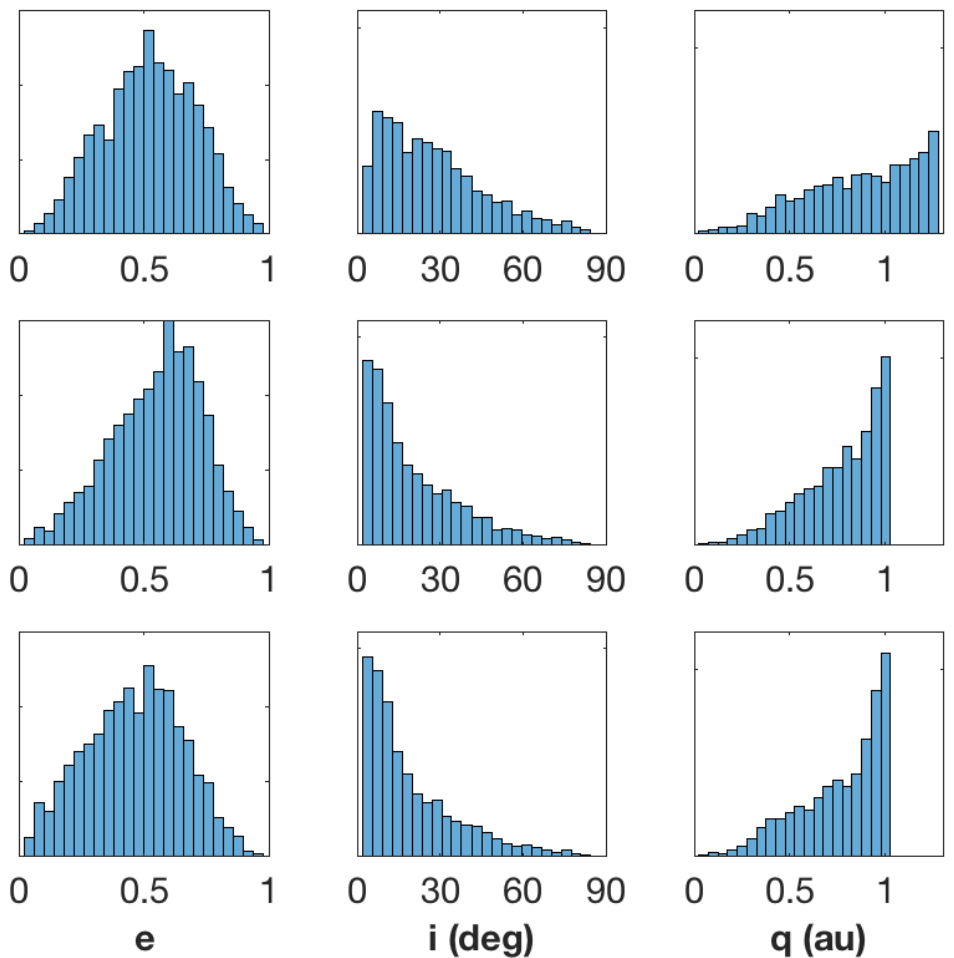

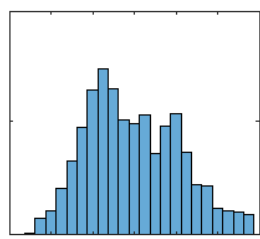

012345

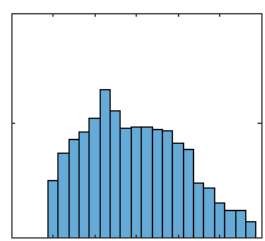

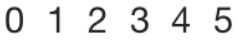

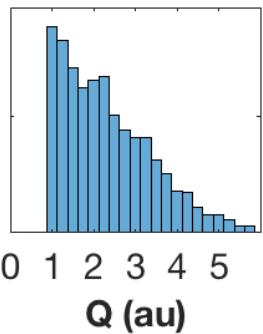

Figure 4. Distribution of orbital elements for NEOs, PHAs and impactors.

we target an impact with a high-fidelity, 11-body dynamical model of the solar system (Sun, eight planets, Moon and Pluto). The year of impact is arbitrary, but the date is fixed by the longitude of the asteroid's impacting node (be it ascending or descending), thus it is straightforward to compute the preliminary epoch of perihelion $t_{p}$. We preserve the value of $\xi^{*}$ obtained in Step 1 and select a random value of $\zeta=\zeta^{*}$ distributed uniformly along the chord for which $\xi=\xi^{*}$ and $b<b_{\oplus}$.

Given the starting element set $E^{0}$, which takes the form $\left(a, e, t_{p}, i, \Omega, \omega\right)$, we target the desired $b$-plane coordinates $\left(\xi^{*}, \zeta^{*}\right)$ with a traditional shooting method informed by the partial derivatives $\partial(\xi, \zeta) / \partial E$, leading to our final impactor elements $E^{*}$. We select an epoch of osculation approximately 30 days prior to the impact so that the osculating elements will not be significantly affected by the Earth's gravity. We also propagate from the year of impact to the epoch 2000-0101.5 TDB to obtain a second element set at a common time for all impactors. With the impact elements in hand we also obtain the impact circumstances for each impactor, including the impact time, location, velocity, and direction.

\section{Properties of the IMPACTING POPUlation}

As a preliminary exploration for this project we have derived a set of 3000 debiased impactor orbits based on the orbital distribution of the smallest size bin of the Granvik NEO model. Roughly 182 million NEOs were sampled in the process of obtaining these 3000 impactors. In this section we explore the properties of this population and put it in context with the larger NEO and PHA populations.

Figure 4 depicts the distributions of selected orbital elements for the Granvik NEO population, for the PHA population (recalling that LMAs are a suitable proxy), and for the impactor population. We make the following observations regarding these distributions:

- The impactors favor lower semimajor axes, which allow more opportunities to impact due to the shorter orbital period. Moreover, the preference for low relative velocities on crossing orbits also weights semimajor axis towards $\sim 1 \mathrm{au}$. - Relative to NEAs, the impactors show a clear trend towards lower eccentricity. This is a result of the lower relative velocities and hence larger capture cross-section at lower eccentricities.

- At low enough inclinations almost every Earth-crossing orbit will be a PHA, and so low inclination orbits are more prevalent among the PHAs. And low inclination is enhanced even more among the impactors due to the lower $V_{\infty}$, which leads to larger $b_{\oplus}$.

- Obviously, an impact is not possible if the asteroid perihelion is beyond the Earth aphelion, nor if the asteroid aphelion is interior to the Earth perihelion. However, those that barely cross these boundaries, known as shallow-crossing orbits, lead to larger impact cross-sections and so impactors have an increased density near 1 au for both perihelion and aphelion distances. There is a significant paucity of PHAs near $Q=1$ au because there are few NEOs interior to Earth, and yet there is a clear excess of impactors with $Q$ near $1 \mathrm{au}$.

As we have mentioned, impactors tend to lower $V_{\infty}$ because of the resulting increase in the impact cross-section on the $b$-plane. Figure 5 depicts the cumulative distribution of both $V_{\infty}$ and $V_{\text {imp }}$ for both the PHA and impactor populations. Table 1 indicates the values at various percentiles for the curves shown in Fig. 5. The median $V_{\infty}$ is $\sim 3 \mathrm{~km} / \mathrm{sec}$ slower for impactors as compared to PHAs, and this translates into slower impact velocities and $\sim 25 \%$ less impact energy compared to the velocity distribution of the PHA population. 


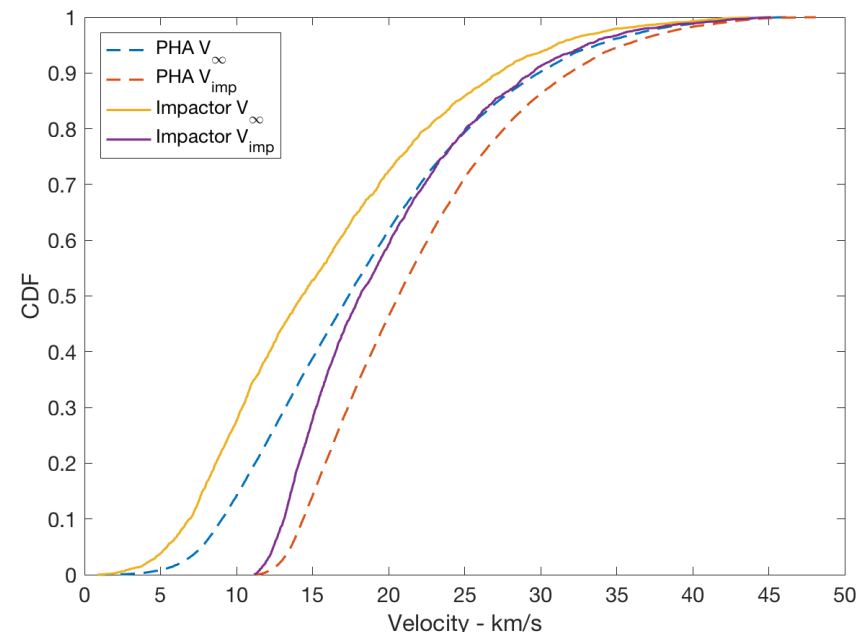

Figure 5. Cumulative distribution of hyperbolic excess velocity $V_{\infty}$ and impact velocity $V_{\text {imp }}$ for both PHAs and the debiased synthetic impactor population.

Table 1. Comparison of the velocity distribution between PHAs and impactors.

\begin{tabular}{cccccc} 
& \multicolumn{2}{c}{$V_{\infty}(\mathrm{km} / \mathrm{s})-$} & & \multicolumn{2}{c}{$V_{\text {imp }}(\mathrm{km} / \mathrm{s})-$} \\
\cline { 2 - 3 } Percentile & PHA & Impactor & & PHA & Impactor \\
\hline 10 & 9.1 & 6.9 & 14.4 & 13.1 \\
50 & 17.4 & 14.3 & 20.7 & 18.1 \\
90 & 29.9 & 27.2 & 31.9 & 29.4 \\
\hline
\end{tabular}

We close this section by examining the distribution of impact location across the Earth's surface. Figure 6 depicts a map of the Earth with the 3000 impact locations superimposed. Because the projection is equal area, any significant nonuniformities in the surface distributions of impacts would be apparent on visual inspection. A more precise examination of this question is shown in Fig. 7, where the distribution in latitude and longitude for the impactors is compared to that obtained for a theoretical uniform area-wise distribution. From this figure it is clear that there is no significant difference between the modeled and theoretical distribution of impact locations on the Earth.

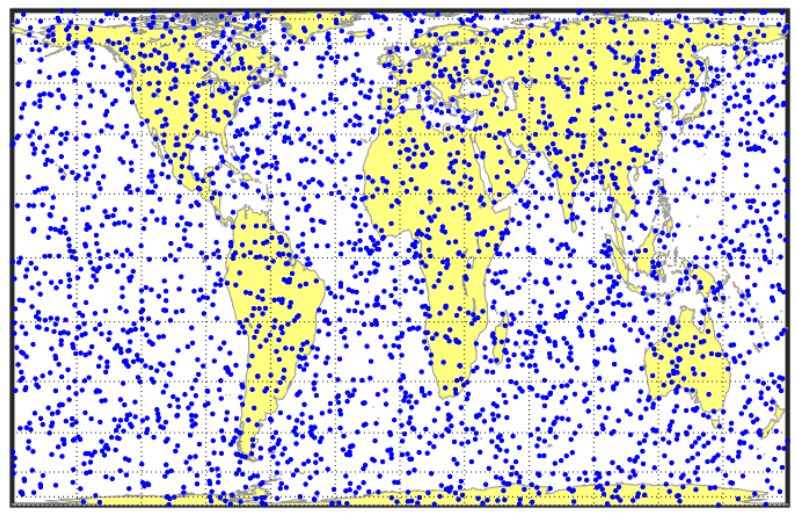

Figure 6. Equal area projection map of the Earth showing the uniform distribution of impactors across the Earth's surface.
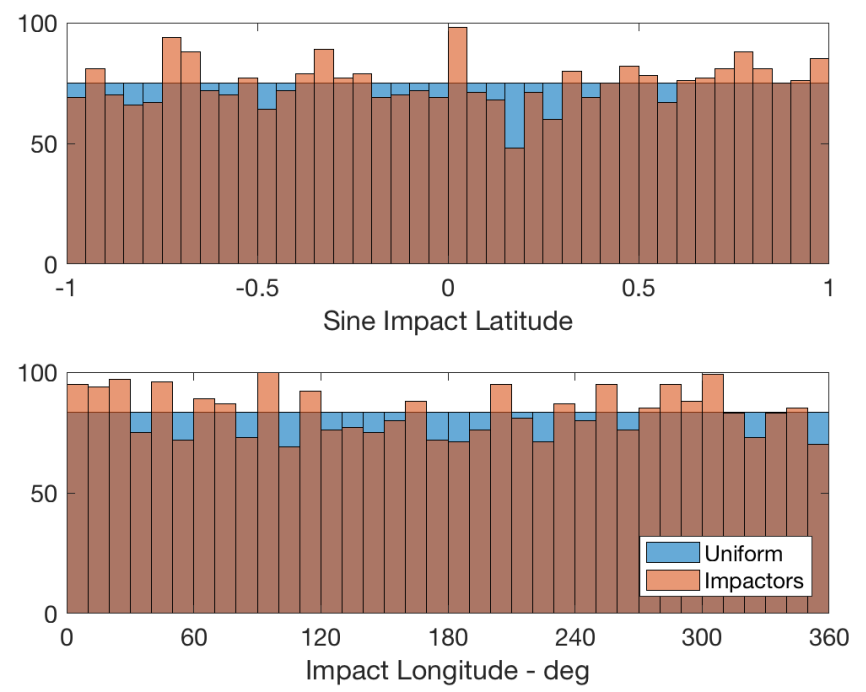

Figure 7. Histogram of sine of latitude (top) and longitude (bottom) of impactors in comparison with a uniform areawise distribution.

\section{Conclusion}

We have produced a debiased set of synthetic Earthimpacting asteroid orbits. We select impactors from among the NEO population according to the annualized impact probability computed under a two-body formalism, and we validate the approach with a Monte Carlo test. The resulting impactor population significantly favors low $V_{\infty}$ orbits due to the increased impact cross-section for such orbits. This leads to an increased presence of low eccentricities and low inclinations among the impactors, as well as an enhancement of orbits having semimajor axis, perihelion distance or aphelion distance near $1 \mathrm{au}$.

As this project comes to completion we intend to publish our synthetic impactor population, and at a number of different size ranges. This will allow other researchers to explore a number of important questions, such as how well NEO surveys are doing at discovering impactors and how they can be tuned to maximize reduction of the impact hazard from undiscovered objects. Other useful studies include evaluation of the impact warning time afforded by surveys and understanding the extent to which impactor properties depend on size or origin.

\section{ACKNOWLEDgments}

This work was conducted in part at the Jet Propulsion Laboratory, California Institute of Technology under a contract with the National Aeronautics and Space Administration. 


\section{REFERENCES}

[1] S. R. Chesley and T. B. Spahr, "Earth impactors: orbital characteristics and warning times," in Mitigation of Hazardous Comets and Asteroids, 2004, pp. 22-37.

[2] T. Grav, R. Jedicke, L. Denneau, S. Chesley, M. J. Holman, and T. B. Spahr, "The Pan-STARRS Synthetic Solar System Model: A Tool for Testing and Efficiency Determination of the Moving Object Processing System," PASP, vol. 123, p. 423, Apr. 2011.

[3] W. F. Bottke, R. Jedicke, A. Morbidelli, J. Petit, and B. Gladman, "Understanding the Distribution of NearEarth Asteroids," Science, vol. 288, pp. 2190-2194, Jun. 2000.

[4] M. Granvik, A. Morbidelli, R. Jedicke, B. Bolin, W. F. Bottke, E. Beshore, D. Vokrouhlický, D. Nesvorný, and P. Michel, "Debiased orbit and absolute-magnitude distributions for near-Earth objects," Icarus, vol. 312, pp. 181-207, Sep. 2018.

[5] E. J. Öpik, Interplanetary Encounters: Close-range Gravitational Interactions. New York: Elsevier Scientific Pub. Co., 1976.

[6] G. B. Valsecchi, A. Milani, G. F. Gronchi, and S. R. Chesley, "Resonant returns to close approaches: Analytical theory," A\&A, vol. 408, pp. 1179-1196, Sep. 2003.

\section{BIOGRAPHY}

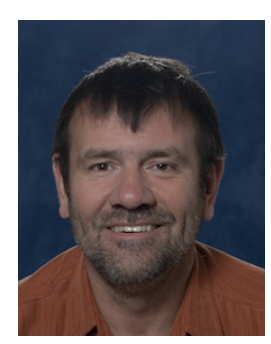

Steve Chesley received his B.S. and M.S. degrees in Aerospace Engineering from Texas A\&M University in 1986 and 1993, and his Ph.D. in Aerospace Engineering from the University of Texas at Austin in 1998. He is currently a Senior Research Scientist in the Solar System Dynamics Group at NASA's Jet Propulsion Laboratory, where he led the development of Sentry, JPL's automated asteroid impact monitoring system. His long-term research focus is on impact hazard assessment and precision orbit determination and ephemeris prediction for small solar system bodies.

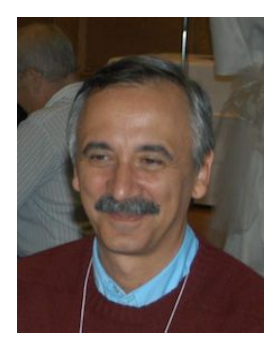

Giovanni B. Valsecchi specializes in the dynamics of Solar System small objects at the Institute of Astrophysics and Space Planetology, a part of the Italian National Institute for Astrophysics, and at the Institute of Applied Physics, which is a part of the Italian National Research Council. His research interests include the various aspects of the chaotic motion of comets and asteroids induced by close planetary encounters, especially the geometric aspects of the problem. He has also studied criteria to assign meteors to streams, the collective motions of space debris after catastrophic fragmentations, and the existence of long duration periodic orbits near the orbit of the Moon.

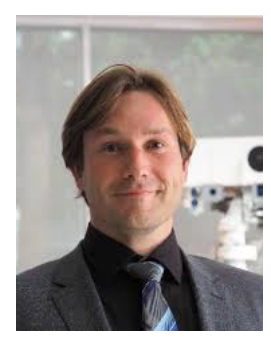

Siegfried Eggl started his career as a lecturer and graduate researcher at the University of Vienna, Austria. After completing his Ph.D. in Astrophysics in 2013, Dr. Eggl moved to Paris, France. At the Institute for Celestial Mechanics and Ephemeris Calculation of the Paris Observatory (IMCCE) he co-developed the current European planetary defense strategy and designed manifold based trajectories as a contractor for Airbus Defense and Space. In 2016, Dr. Eggl accepted an appointment at JPL with the Solar System Dynamics group to continue his research on asteroid orbit deflection. Since 2018 he is a research associate at the University of Washington working on the Moving Object Processing System of the Large Synoptic Survey Telescope (LSST).

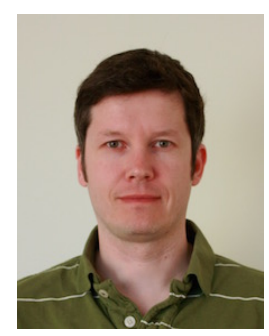

Mikael Granvik is an Associate Professor at Luleå University of Technology in Sweden and an Academy Research Fellow at the University of Helsinki in Finland. He received a Ph.D. in Astronomy from the University of Helsinki in 2008 and held a postdoctoral position at University of Hawaii where he worked on the Pan-STARRS survey. He is an expert in NEO population models as well as topics related to asteroid orbit computation such as impact-probability estimation for imminent impactors. Asteroid (14328) Granvik is named after him.

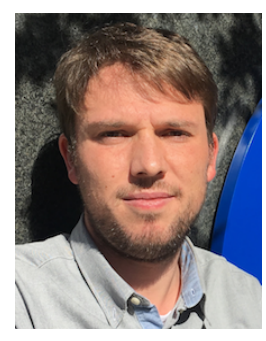

Davide Farnocchia received his B.S. and M.S. degrees in Mathematics from the University of Pisa in 2006 and 2008, and a Ph.D. in Mathematics in 2012. He currently is a Navigation Engineer at NASA's Jet Propulsion Labroatory. His research activities include trajectory estimation and impact hazard assessment for small bodies of the Solar System such as asteroids and comets.

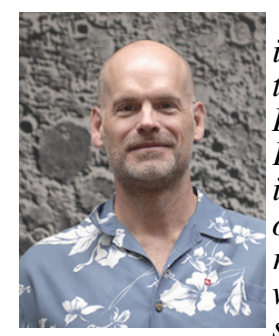

Robert Jedicke received his PhD in experimental particle physics from the University of Toronto, Canada. He held post-doctoral positions at the Fermi National Accelerator Laboratory in Batavia, Illinois, and at the University of Arizona's Lunar \& Planetary Laboratory where he worked on the Spacewatch near-Earth asteroid survey. He spent more than five years as a software engineer at Veeco Corporation in Tucson, Arizona, before accepting a faculty position at the University of Hawaii's Institute for Astronomy where he was the development manager of their Moving Object Processing System for the PanSTARRS telescope. 